\title{
THINK SIMULATION - THINK EXPERIMENT: THE VIRTUAL CELL PARADIGM
}

\author{
Ion I. Moraru \\ James C. Schaff \\ Leslie M. Loew \\ Dept. of Cell Biology \\ University of Connecticut Health Center \\ Farmington, CT 06030-3505, U.S.A.
}

\begin{abstract}
The Virtual Cell modeling and simulation framework is the product of interdisciplinary research in biology that applies the diverse strengths and experiences of individuals from engineering, the physical sciences, the biological sciences, and mathematics. A key feature is the separation of layers (core technologies and abstractions) representing biological models, physical mechanisms, geometry, mathematical models and numerical methods. This reduces software complexity, allowing independent development and verification, but most importantly it clarifies the impact of modeling decisions, assumptions, and approximations. The result is a physically consistent, mathematically rigorous, spatial modeling and simulation framework for cell biology. The Virtual Cell has a rich, interactive user interface which connects to remote services providing scalable access to a modeling database and a large dedicated cluster for shared computation and storage. In addition to new modeling capabilities, future developments will emphasize data and tool interoperability, extensibility, and experimentally oriented model analysis tools.
\end{abstract}

\section{INTRODUCTION}

The accelerating progress in cataloguing the critical molecular and structural elements responsible for cell function has led to the hope that cell biological processes can be analyzed and understood in terms of the interactions of these components. Besides the obvious need for the acquisition and organization of quantitative data on these interactions, another critical prerequisite for such analyses is the effective synthesis of this data by constructing models that can then predict the overall behavior of the biological system. If the model correctly predicts the biological endpoint, one can hypothesize that the elements within the model are sufficient, and often one can discern which of these elements are the most critical. This can then be tested by further experiments designed to specifically perturb or remove these elements (e.g., gene knockouts, drug targeting). Perhaps more useful, however, is when the model is unable to predict the observed biology, implying that the elements of the model are either incorrect or incomplete. Analysis of such faulty models can directly motivate the discovery, via new experiments, of previously unknown critical biochemical or structural features required for the cellular process under investigation.

However, the difficulties associated with the formulation of mathematical models and the generation of simulations from them has impeded the adoption of this disciplined and quantitative approach to research in traditional cell biology, as well as slowed down the process of realizing the ultimate potential of the new, large scale, "omic" datasets, keeping the rapidly growing area of systems biology at a level of mostly qualitative, top-down approaches (Moraru and Loew 2005). Because biologists rarely have sufficient training in the mathematics and physics required to build quantitative models, modeling has been largely the purview of theoreticians who have the appropriate training but little experience in the laboratory. With the notable exception of areas with rich traditions of biophysical research (such as cardiac and neuronal electrophysiology), this disconnection to the laboratory has limited the impact of mathematical modeling in cell biology and, in some quarters, has even given modeling a poor reputation.

\section{ABSTRACTING BIOLOGY FROM SIMULATION}

The Virtual Cell project (<http://vcell.org/>) aims to address the above problems by providing a computational modeling framework that is accessible to cell biologists. It does this by abstracting and automating the mathematical and physical operations involved in constructing models and generating simulations from them. It was originally developed to specifically target the need for building and solving spatial models of intracellular phenomena, and it has since grown into a large platform that is being continuously enhanced with new features (Slep- 
chenko et al. 2003). The current layered, modular design of the Virtual Cell was motivated by several factors: managing complexity, facilitating model reuse, providing biologically and mathematically oriented tools, and building a distributed architecture.

One of the innovative features in the design of the Virtual Cell is the introduction of three distinct layers of abstractions in the modeling process, which are following a paradigm similar to hypothesis-driven experimental studies. Figure 1 schematizes the way in which the modeling process is structured within the Virtual Cell. This hierarchical layered structure starts with the system Physiology essentially the mechanistic hypothesis to be investigated. It includes the description of the molecular species and hypothesized interactions localized to cellular structures. Cellular structures need only be specified as the topological arrangement of membranes and membrane bounded compartments. Interactions can be biochemical reactions defined either within volumetric compartments of the cell or in membranes; molecular fluxes across membranes; and electrical currents. Additionally, the Physiology captures the biochemical and biophysical properties of these interactions as rate laws for reactions and fluxes rate (that can be determined as an explicit function of the local environment, e.g., concentrations, surface densities, membrane potential, and of one or more kinetic parameters, e.g., $\mathrm{k}_{\mathrm{on}}$ and $\mathrm{k}_{\text {off }}$ ). Mass action and Michaelis-Menten rate laws are available automatically, and arbitrary user-defined general kinetic expressions can also be used. Membrane transport kinetics can be specified with expressions for molecular flux or, for ions, the electric current. The transport kinetics can be described in terms of standard electrophysiological formulas (e.g., Goldman-Hodgkin-Katz permeability or Nernst conductance) or as user defined molecular flux or current.

The Physiology can then have several Applications essentially the "virtual experiment" environment, the set of conditions under which one or more simulations will be performed. A particular Application would specify a specific geometry (e.g., size and shape of a cell), the boundary conditions, default initial concentrations and parameter values, and whether any of the reactions are sufficiently fast to permit a pseudo-steady state approximation. Also at the Application level, individual reactions can be disabled as an aid in determining the proper initial conditions for a prestimulus stable state. An Application of a Physiology is sufficient to completely describe the governing mathematics of the model and the mathematical representation of the system is automatically generated at this point by the software (stored in a proprietary, but quite intuitive, mathematical description language, VCMDL, that can be viewed by the user). The Virtual Cell is designed to maintain a separation between this mathematical description, generated either via a BioModel or a MathModel, and the details of how the simulations are implemented.

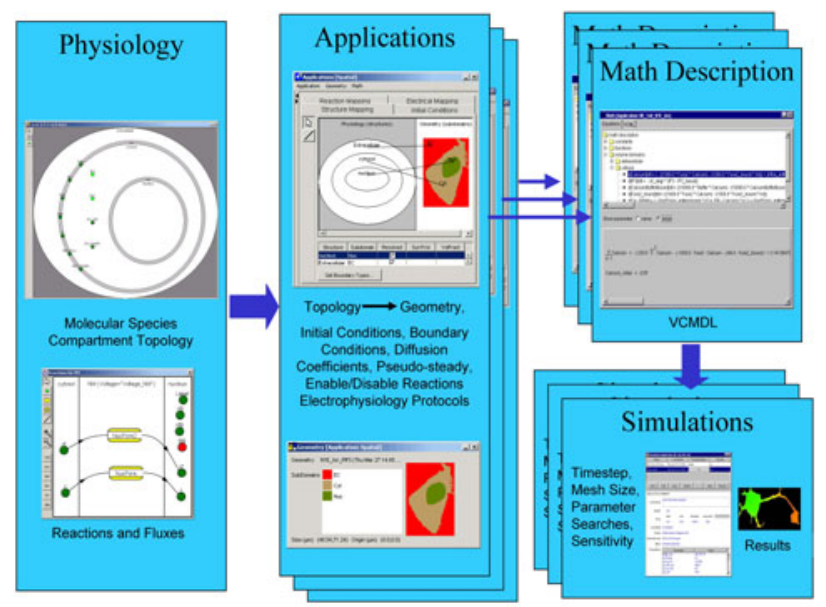

Figure 1: Decomposing the Modeling Process in the Virtual Cell BioModel Workspace

Finally, as shown in Figure 1, several simulations can be spawned off of a given Application. That is, the mathematics can be solved with multiple choices of numerical solver, time step, mesh size for spatial simulations, time duration and overrides of the default initial conditions or parameter values to permit parameter scans. A local sensitivity analysis service is also available at the simulation level to aid in parameter estimation and to determine which features of the model are most critical in determining its overall behavior. A parameter estimation tool can fit nonspatial models to time series experimental data. A particular simulation specification is, in turn, sufficient to describe the software requirements for numerically calculating solutions and the Virtual Cell thus automatically generates the appropriate $\mathrm{C}++$ code to produce simulation results.

Such a decomposition of modeling concepts makes possible the effective reuse of the model in multiple contexts. To allow a single quantitative mechanistic hypothesis to be validated under multiple experimental conditions at different scales and using different physical approximations, the abstract physiological modeling concept is utilized. For example, a receptor binding reaction localized to a membrane can have many different mathematical forms while the quantitative mechanistic description is unchanged. Its form depends on whether the membrane is mapped to a spatially resolved surface, or mapped volumetrically as a distributed surface for an unresolved organelle, or as non-spatial representation in a compartmental model. The mathematical form also depends on additional modeling assumptions, such as whether the reaction is "knocked out" or considered to be "fast" (pseudo-steady state approximation).

We will now use a study of calcium dynamics in a neuronal cell (Fink et al. 1999, Fink et al. 2000) as a concrete illustration of this paradigm. That study started from the experimental observation that the neuromodulator bra- 
dykinin applied to the cells produced a calcium wave that starts in the neurite and spreads to the soma and growth cones (the calcium wave was recorded by digital microscope imaging of a fluorescent calcium indicator). The hypothesis was that interaction of bradykinin with its receptor on the plasma membrane activated production of inositol1,4,5-trisphosphate $\left(\mathrm{InsP}_{3}\right)$ that diffused to its receptor on the endoplasmic reticulum (ER) leading to calcium release. A model was constructed in the Virtual Cell software, where the Physiology component contained the biochemical interactions decribing the kinetics of $\mathrm{Ins}_{3}$ production, the transport of calcium through the $\mathrm{InsP}_{3}$ receptor calcium channel, the SERCA pump activity, and calcium buffering (all based on direct experimental studies, new or from existing literature). At the Application level, the specifics of the calcium wave experiments were then implemented: the actual geometry to be used for simulations was created from real microscope images of the cultured cells, details of the relevant receptor distributions were added (obtained from quantitative immunofluorescence experiments), and initial conditions were specified (some directly from experimental measurements, other, unknown, from separate compartmental simulations that were run until a steadystate equilibrium was reached), as well as parameters relevant to the experimental stimulus. Several Applications were spawned off to test individual experimental conditions, and several simulations in each Application were defined and run to test for varying time courses and parameter values. The mathematical equations generated by every particular combination of hypothesis, experimental conditions, and parameter values, were then solved to produce a simulation of the spatiotemporal pattern of calcium that could be directly compared to the experiment. The power of this approach is evident once one realizes that all the different Applications and Simulations were reusing the same mechanistic hypothesis of the intracellular interaction network controlling calcium dynamics described in the Physiology. On the other hand, when some of the elements of the mechanistic hypothesis were being questioned, and corresponding changes were being made to the Physiology, they were seamlessly propagated to all Applications and their Simulations, which could simply be rerun with a push of a button under the new hypothesis, all the while preserving the details of the experimental conditions to be simulated.

This example is also useful to illustrate the conceptual aspects of applying the scientific method in a quantitative cell biology modeling study. Building a biological model in the Virtual Cell is an iterative process that should start with a simple model that is incrementally validated against simulations and experimental observations. In our calcium dynamics study, for example, an initial assumption was that the InsP3 receptor was distributed uniformly throughout the cell. The simulations produced under this assumption gave calcium amplitudes that were much too high in the neurite and too low in the soma compared to the experimental calcium dynamics. This prompted us to investigate the $\mathrm{InsP}_{3}$ receptor distribution by immunofluorescence revealing that the density in the soma was twice as high as the neurite; the simulation resulting from this new elaboration of the model provided an excellent match to the experimental calcium wave. Furthermore, a model (or hypothesis) should lead to predictions that can be further tested with new experiments. For example, our model predicted that if bradykinin were applied to different regions of the cell, rather than globally, application to the proximal neurite would be sufficient to produce a calcium wave; but activation of bradykinin receptors in only the growth cone or soma was predicted to produce an aborted calcium wave. These predictions were subsequently borne out by experiments in which bradykinin was regionally applied via pressure application through a micropipet (Fink et al. 1999).

\section{REACTION/DIFFUSION, FLOW AND ELECTRODIFFUSION IN ARBITRARY GEOMETRIES}

The design approach to the Virtual Cell client interface described in the previous section also inspired and facilitated the creation of an overall modular software structure, that allows independent development and validation of individual services, which are loosely coupled in an understandable overall architecture. For example, the solvers only know mathematics, not physics or biology. This allows a progressive introduction of new capabilities, starting with development and validation of numerical tools that are designed to handle certain new classes of mathematical problems (e.g., partial and ordinary differential equations of certain form as described below). When the supported class of mathematics is expanded, this new capability is instantly available to a mathematically oriented user. The richer mathematical form then provides new flexibility for mapping physiological mechanisms and geometry to a concrete mathematical form required for simulation.

At its most fundamental level, a cell biological process can be described as the consequence of a complex series of chemical transformations. To understand the process, the relevant molecules have to be identified and their timevarying concentrations and spatial distributions have to be determined. A model, at this molecular level, chooses all the presumed chemical species, assigns them initial concentrations and spatial distributions and connects them with appropriate kinetic expressions. A simulation that predicts the spatiotemporal behavior of this system has to solve a class of problems known as reaction/diffusion equations. The mathematical problem is summarized by the equations:

$$
F_{i}=-D_{i} \nabla C_{i}-z_{i} \mu_{i} C_{i} \nabla \Phi-C_{i} V_{i} \quad \mu_{i}=\frac{D_{i} F}{R T}
$$




$$
\begin{gathered}
R_{i}=\frac{d[i]}{d t}=k_{1}[k][j]-k_{-1}[i] \\
\frac{\partial C_{i}}{\partial t}=-\operatorname{div} F_{i}+R_{i}
\end{gathered}
$$

The first line is a modified form of the familiar NernstPlanck equation that describes the flux, $F_{i}$, of a molecule i, driven by its concentration gradient, $\nabla C_{i}$, and, if it has an ionic charge $z_{i}$, the electric field in the system $\nabla \Phi$. The diffusion coefficient, $D_{i}$, and the mobility $\mu_{i}$, are the proportionality constants for these driving forces. The Virtual Cell has recently added the capability of accounting for flow, as indicated by the term $C_{i} V_{i}$, where $V_{i}$ is the velocity of molecular species $i$. The second line portrays a typical reaction that produces molecule $\mathrm{i}$ (while consuming $\mathrm{j}$ and $\mathrm{k})$. The mass action ordinary differential equation for the rate of change of $\mathrm{i}, R_{i}$, depends on the concentrations of the reactants and products. In general, $R_{i}$ can depend on the concentrations of any of the molecules in the system and may have a more complex form than the mass action expression shown here. The third line combines the fluxes and reactions into a system of partial differential equations that must be integrated to simulate the behavior of the molecular species.

The fact that the Virtual Cell is designed to handle any reaction system in any geometry, precludes the formulation of a general analytical solution for the problem. There are two generic approaches to numerical solutions - stochastic and continuous. The continuous approach provides a deterministic description in terms of average species concentration. This approach is effective and accurate so long as the number of molecules in a system is large, such that thermal stochastic fluctuations around average values can be ignored. We have found that the finite volume method (Patankar 1980) for discretization of a system of PDEs is especially well suited for our problem domain - that is reaction/diffusion equations in arbitrary geometries (Choi et al. 1999, Schaff et al. 1997, Schaff et al. 2001). Of course, the software can also solve non-spatial problems corresponding to systems of ODEs describing reactions within well stirred compartments and fluxes across the membranes that separate the compartments. The software provides a choice of several solvers for such compartmental problems including a stiff solver. For both spatial and compartmental problems, we have implemented an automated pseudo-steady approximation that can be invoked by the user when a subsystem of reactions equilibrates rapidly on the timescale of the overall process of interest (Slepchenko et al. 2000).

The current version of Virtual Cell handles only continuous solutions, and alternate stochastic solutions are currently in the development and testing phase. Stochastic fluctuations can become important if the number of molecules involved in a process is relatively small. For fully stochastic problems in which the number of particles in a reaction/diffusion system is too small to solve with numerical solutions of PDEs, diffusion can be described as Brownian random walks of individual particles and chemical kinetics is simulated as stochastic reaction events. We also need to consider hybrid systems of stochastic differential equations where one can combine the numerical techniques commonly applied to regular differential equations and Monte Carlo methods employing random number generators. We have recently prototyped an efficient algorithm in which the probabilities of each reaction are calculated from rate constants and numbers of substrate molecules (Gillespie 1977, 2001). A stochastic method is used to determine which reaction will occur based on their relative probabilities. The time step is then adjusted to match the particular reaction that occurs. After the reaction is complete the numbers of substrate molecules are readjusted prior to the next cycle. We have also prototyped a module to simulate Brownian dynamics of particles in arbitrary geometries where the particles can react with continuously distributed species. Although these approaches have been implemented in our $\mathrm{C}++$ library and have been applied to problems on the dynamics of RNA granule trafficking (Carson et al. 2001), generalized stochastic modeling capabilities are not yet fully developed. A particular challenge is the efficient computation of models that consider rare events involving few molecules in the presence of very frequent events among a large population of molecules.

\section{VIRTUAL CELL SOFTWARE COMPONENTS}

The Virtual Cell is a continuously evolving, large-scale software project where complexity has been managed by decomposing the software into a collection of loosely coupled services interacting with conceptually simple data abstractions, which is critical for the robustness and flexibility of the distributed architecture of the platform (remote clients/database server/compute nodes/simulation data storage, etc.) illustrated in Figure 2.

The server-side components were originally deployed as RMI servers (Java Remote Message Interface) with the appropriate RMI protocol wrappers. New protocol wrappers were developed to adapt these services to an architecture based on a message-oriented middleware solution (components became asynchronous, stateless, and transactional). The Database Service (which internally consists of multiple database layers) provides object-relational mapping, object caching, transactions, connection management, and object access control. The component interface consists of simple object persistence operations such as load, save, update, delete, annotate, and query. The Simulation Data Service provides simulation results retrieval, 
caching, data reduction, exporting and access control. The Simulation Dispatch Service provides batch scheduling, enforces quotas, monitors running simulations with checkpointing and restarting and load balancing. The Simulation Worker Service normally runs on the compute nodes of our cluster and is responsible for code generation, compilation, running the simulations, reporting progress, and storing simulation results to the file server.

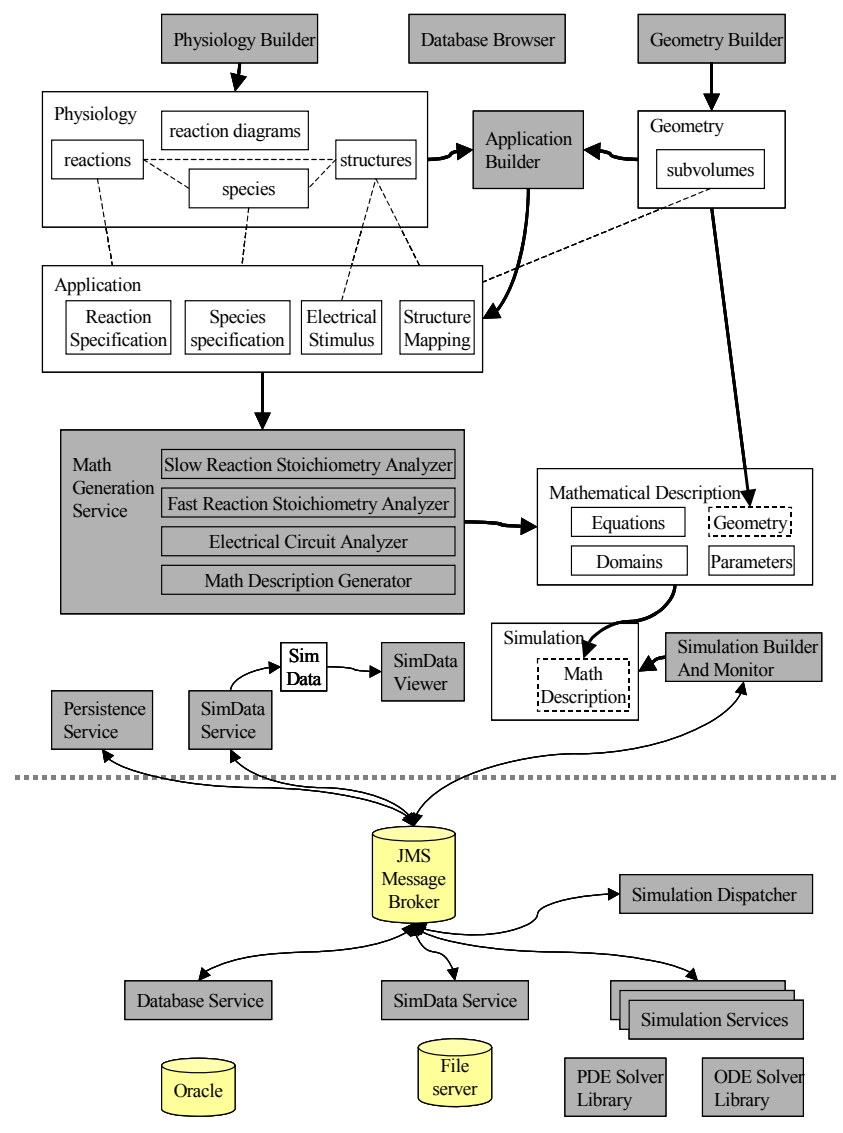

Figure 2: Existing Virtual Cell Software Components

All of these services are capable of being deployed (without the protocol wrappers) in a standalone application context. A Math Generation service was originally deployed as a server-side service but is now deployed locally on the client. This service generates a declarative Mathematical Description from an Application. This transformation is invoked before saving or running a simulation, and serves to decouple the biological representation from the mathematical representation. There were also client-side components developed to simplify the user interface design by decoupling the user interface components (editors, etc.) from remote services and any data management issues.

\section{EXCHANGES WITH EXTERNAL RESOURCES}

The Virtual Cell provides mechanisms to link to external resources to facilitate the sharing of models across various computational cell biology and systems biology tools. This is achieved by supporting the 2 prevalent XML languages that cover these fields: SBML (<http://WwW . sbml.org/ $>$ ) and CellML (<http://www. cellml.org/>). The Virtual Cell team has been involved in development of SBML since its inception. Exporting or importing SBML or CellML documents within Virtual Cell is simply achieved through appropriate menu items under the File menu. This facilitates the exchange of VC model data between complementary software systems as over 90 different tools now support at least one of these languages at varying levels. However, neither of these languages currently support spatial modeling and they have limited support for the abstractions used in Virtual Cell for membrane transport. Also, SBML does not support electrophysiological phenomena. So there was a need to develop an XML dialect that could completely capture a VC model and, accordingly, VCML was developed to meet this need. In addition to allowing ready transfer and documentation of VC models outside our central database, VCML is used internally, for efficient transport of model elements between software modules.

Virtual Cell also has provided access to external databases as a means to strengthen the utility of our own internal $\mathrm{db}$ and to provide additional modeling resources and data to users. To define a molecular species unambiguously, we have provided the ability to bind to a dictionary of species derived from KEGG (their Compound database for small molecules, metabolites, lipids, etc. and their Enzyme database for enzymes) and SwissProt (for proteins). This also allows the user to search his/her own models ('My Models') or other public VC models ('All Models') for molecules that have already been bound. Virtual Cell also imports data from external databases to automate the process of defining and binding new species and generating fragments of reaction schemes. During this import process, in addition to inserting this enzymatic reaction, all the newly created species are automatically bound to the respective external database definitions.

\section{CONCLUSION}

We have described above the design principles and overall structure of the Virtual Cell, a unique software platform for modeling and simulation in the field of cell biology, which is based on a paradigm similar to the classical scientific method applied in experimental, "wet lab" studies. This approach, combined with features specifically targeting experimentalists (intuitive biological user interface, automatic math generation), with the fact that it is freely accessible via the web, does not require specialized local soft- 
ware or hardware resources, and allows collaborative work, has led to an increasing popularity among cell biologists. There are currently hundreds of active users and numerous papers have been recently published describing combined experimental and theoretical studies using the Virtual Cell, addressing a quite wide variety of cell biological processes, such as intracellular calcium dynamics (Fink et al. 1999, 2000, Xu et al. 2003, Fridlyand et al. 2003, Hernjak et al. 2005), nuclear transport (Smith et al. 2002, Kalab et al. 2006), membrane electrophysiology (Suh et al. 2004, Horowitz et al. 2005), cell cycle regulation (Slepchenko and Terasaki 2003), gradient sensing mechanisms (Ma et al. 2004, Schneider and Haugh 2005), etc.

Last but not least, since being made publicly available six years ago, the Virtual Cell framework has been continuously enhanced, and many new capabilities are currently under development, such as new solvers (stochastic and hybrid), new model analysis features (parameter scanning and optimization), new Application tools for explicit representation of experimental data, protocols and virtual instruments, new abstractions for encapsulation of model components and hierarchical assembly of large models, and more. An up-to-date list of features as well as links to public models and published papers is available at the Virtual Cell homepage (<http://vcell.org/>), which also provides access to running the latest version of the software.

\section{ACKNOWLEDGMENTS}

This work was supported by NIH grants P41-RR13186, U54-RR022232, P20-GM65764.

\section{REFERENCES}

Carson, J. H., H. Cui, W. Krueger, B. Slepchenko, B. Brumwell, and E. Barbarese. 2001. RNA trafficking in oligodendrocytes. In Cell Polarity and Subcellular Localization. D. Richter, ed. Springer-Verlag, Berlin.

Choi, Y. S., D. Resasco, J. Schaff, and B. Slepchenko. 1999. Electro-diffusion of ions inside living cells. IMA Journal of Applied Mathematics 62:207-226.

Fink, C. C., B. Slepchenko, I. I. Moraru, J. Schaff, J. Watras, and L.M. Loew. 1999. Morphological control of inositol 1,4,5-trisphosphate signals. Journal of Cell Biology 147:929-935.

Fink, C. C., B. Slepchenko, I. I. Moraru, J. Watras, J. C. Schaff, and L.M. Loew. 2000. An image-based model of calcium waves in differentiated neuroblastoma cells. Biophysical Journal 79:163-183.

Fridlyand, L. E., N. Tamarina, and L. H. Philipson. 2003. Modeling $\mathrm{Ca}^{2+}$ flux in pancreatic B-cells: role of the plasma membrane and intracellular stores. American
Journal of Physiology - Endocrinology and Metabolism 285: E138-154.

Gillespie, D. T. 1977. Exact stochastic simulation of coupled chemical reactions. Journal of Physical Chemistry 81:2340-2361.

Gillespie, D. T. 2001. Approximate accelerated stochastic simulation of chemically reacting systems. Journal of Chemical Physics 115:1716-1733.

Schneider, I. C., and J. M. Haugh. 2005. Quantitative elucidation of a distinct spatial gradient-sensing mechanism in fibroblasts. Journal of Cell Biology 171:883892.

Hernjak, N., B. M. Slepchenko, K. Fernald, C. C. Fink, D. Fortin, I. I. Moraru, J. Watras, and L. M. Loew. 2005. Modeling and analysis of calcium signaling events leading to long-term depression in cerebellar Purkinje cells. Biophysical Journal 89:3790-3806.

Horowitz, L. F., W. Hirdes, B. C. Suh, D. W. Hilgemann, K. Mackie, and B. Hille. 2005. Phospholipase C in living cells: activation, inhibition, $\mathrm{Ca}^{2+}$ requirement, and regulation of M current. Journal of General Physiology 126:243-262.

Kalab, P., A. Pralle, E. Y. Isacoff, R. Heald, and K. Weis.. 2006. Analysis of a RanGTP-regulated gradient in mitotic somatic cells. Nature 440: 697-701.

Ma, L., C. Janetopoulos, L. Yang, P. N. Devreotes, and P.A. Iglesias. 2004. Two complementary, local excitation, global inhibition mechanisms acting in parallel can explain the chemoattractant-induced regulation of $\mathrm{PI}(3,4,5) \mathrm{P}_{3}$ response in Dictyostelium cells. Biophysical Journal 87:3764-3774.

Moraru, I. I, and L. M. Loew. 2005. Intracellular signaling: spatial and temporal control. Physiology 20: 169-179.

Schaff, J., C. C. Fink, B. Slepchenko, J. H. Carson, and L. M. Loew. 1997. A general computational framework for modeling cellular structure and function. Biophysical Journal 73:1135-1146.

Schaff, J. C., B. M. Slepchenko, Y. Choi, J. M. Wagner, D. Resasco, and L. M. Loew. 2001. Analysis of nonlinear dynamics on arbitrary geometries with the virtual cell. Chaos 11:115-131.

Patankar, S. V. 1980. Numerical Heat Transfer and Fluid Flow. Washington, D.C.: Taylor and Francis.

Slepchenko, B. M., J. C. Schaff, I. G. Macara, and L. M. Loew. 2003. Quantitative cell biology with the virtual vell. Trends in Cell Biology 13:570-576.

Slepchenko, B. M., and M. Terasaki. 2003. Cyclin aggregation and robustness of bio-switching. Molecular Biology of the Cell 14: 4695-4706.

Smith, A. E., B. M. Slepchenko, J. C. Schaff, L. M. Loew, and I. G. Macara. 2002. Systems analysis of Ran transport. Science 295:488-91.

Suh, B. C., L. R. Horowitz, W. Hirdes, K. Mackie, and B. Hille. 2004. Regulation of KCNQ2/KCNQ3 current by $\mathrm{G}$ protein cycling: the kinetics of receptor-mediated 
signaling by Gq. Journal of General Physiology 123:663-683.

Xu, C., J. Watras, and L. M. Loew. 2003. Kinetic analysis of receptor-activated phosphoinositide turnover. Journal of Cell Biology 161:779-791.

\section{AUTHOR BIOGRAPHIES}

ION I. MORARU is Assistant Professor of Cell Biology at the University of Connecticut Health Center. He is heading the Computational Facility of the Richard D. Berlin Center for Cell Analysis and Modeling. His e-mail address is <moraru@panda. uchc. edu>.

JAMES C. SCHAFF is Assistant Professor of Cell Biology at the University of Connecticut Health Center. He is the lead software developer for the Virtual Cell project. His e-mail address is $<$ schaffeneuron. uchc. edu>.

LESLIE M. LOEW is Professor of Cell Biology and Computer Science at the University of Connecticut Health Center. He is the Director of the Richard D. Berlin Center for Cell Analysis and Modeling, an NIH National Technology Center in Networks and Pathways. His e-mail address is $<$ les@volt. uchc . edu>. 